\title{
Free Terminal Time in Optimal Control approach of Chikungunya Model
}

\author{
Meryem Alkama \\ Laboratory of Analysis \\ Modeling and Simulation
}

\author{
Mostafa Rachik \\ Laboratory of Analysis \\ Modeling and Simulation
}

\begin{abstract}
Optimal control problems are an important mathematic tool used to reduce infectious diseases, most of works in this area considered time constant. In this paper, we present a free terminal optimal time control of Chikungunya epidemic model, which is an arthropod-borne virus (arbovirus) transmitted by mosquitoes of Aedes genus, with the order to give a minimum duration needed to reduce the infected group of both human and vector. We present a control simulating program using Matlab routines. The optimal control and the optimal final time are found using Pontryagin's maximum principle and the additional transversality condition for the terminal time. We solved the optimality system by an iterative method, then we confirm the performance of the optimization strategy by numerical simulations.
\end{abstract}

\section{General Terms}

Optimal conrol, epidemiology, numerical analysis

\section{Keywords}

Opimal control problems, numerical simulations, chikungunya

\section{INTRODUCTION}

Chikungunya is an emerging vector-borne disease of considerable significance and prevalence in Member countries of World Health Organization (WHO) South-East Asia (SEA) Region. The disease has been reported from countries of South and East Africa, South Asia and South-East Asia. In the WHO South-East Asia Region, out-breaks have been reported from India, Indonesia, Myanmar, Sri Lanka, Thailand and Maldives. Massive outbreaks of chikungunya fever have occurred in recent years in India and in the island countries of the Indian Ocean. As of 20 June 2014, 5294 confirmed cases and more than 180000 suspected cases of chikungunya epidemic have been reported from the start of the outbreak in Saint Martin in December 2013 [2].

A major epidemic of this disease was reported in 2005.2006 in Reunion Island; with approximatively 266,000 residents (34.3\% of the population) of this Indian Ocean Island were affected by chikungunya fever as of February 19, 2007. This epidemic also spread to France through imported cases from Reunion Island. Historically, chikungunya was considered self-limiting and nonfatal. However, 254 deaths on Reunion (case-fatality rate $1 / 1,000$ ) that were attributed directly or indirectly to chikungunya during the epidemic changed this perspective $[1,4]$.

Chikungunya is a mosquito-borne viral disease caused by an alphavirus from the Togaviridae family. It is transmitted by the bite of Aedes mosquitoes, primarily Aedes aegypti and Aedes albopictus, which are active during the day [1]. Humans are thought to be the major source, or reservoir, of chikungunya virus for mosquitoes. Therefore, the mosquito usually transmits the disease by biting an infected person and then biting someone else. An infected person cannot spread the infection directly to other persons (i.e. it is not a contagious disease). Aedes aegypti mosquitoes bite during the day time. This mosquito species bites during the daytime as well as at dusk. It has the ability to breed in natural and artificial habitats, such as tyres, barrels, rainwater gulley, catch basins, and drinking troughs [3]. The time between the bite of a mosquito carrying chikungunya virus and the start of symptoms ranges from 1 to 12 days.

There is no specific treatment for chikungunya. Supportive therapy that helps ease symptoms, such as administration of non-steroidal anti-inflammatory drugs, and getting plenty of rest, may be beneficial. Infected persons should be isolated from mosquitoes in as much as possible in order to avoid transmission of infection to other people. The lack of a vaccine or specific treatment, in combination with the presence of Ae. aegypti and Ae. albopictus make preventive measures necessary, using larvicides where the water cannot be removed can also be major method to control the spread of chikungunya disease.

In recent years, mathematical models have become a powerful tool for predicting the developing tendency of the infectious disease, determining the key factors of the spread of infectious disease and seeking the optimum strategies of preventing and controlling the spread of infectious diseases [4]. A number of studies have been conducted to explore optimal control theory in some mathematical models for infectious diseases including HIV diseases [7], tuberculosis [8] and vector-borne diseases [9].

In [5], mathematical models describing the dynamics of vector-borne diseases and transmission taking into account the controlling mechanisms applied on the vector population. It deals with two models involving differential equations for the mosquito population and virus transmission to the human population. In [6] an optimal control of chikungunya disease is developed with the aim to reduce the vector, treat and prevent the disease. Moreover, only the constant time is considered in these works. This work can be seen as a complement to the study of [6] and [5], in which we consider a free terminal time. Using models described in [6] for the mosquito population dynamics and the transmission virus, we formulate the associated control model in order to derive optimal prevention and treatment strategies with minimal implementation cost and in the minimal time. Controls used here are based on three main actions applied in the recent epidemics [6].

The paper is organized as follows. In section 2, we present the compartmental model used in [6] to describe the Aedes albopictus population dynamics and the chikungunya virus transmission to the human population. In section 3 , we formulate a free terminal time optimal control problem with an objective functional that minimizes the duration of both treatment and prevention. We also derive a corresponding 
optimality system, from which the optimal time and control may be obtained, which characterizes the optimal control using Pontryagin.s Maximum Principle [36] in free terminal time version [10]. In section 4 numerical results illustrate our theoretical results.

\section{MODEL FORMULATION}

\subsection{Vector Life Cycle}

The life of the vector consists of four stages: embryonic stage (eggs), larvae stage, pupae stage and adult stage. The first three stages need water for their development while the last one needs only air. In this work, as in [5] we will not distinguish the larvae stage and the pupae stage: these two stages are called the immature stage. The lifespan of each stage depends on several factors, such as temperature or the availability of food and water [11]. The vector population is described by a stage-structured model based on the biological life cycle. It consists in four main stages described by the following compartment: egg (E), larvae and pupae (L) which are biologically much closed stages, and the adult stage (A) which contains only females because they are responsible for the transmission.

- The per capita mortality rate of eggs, larvae and adults are denoted by $\mathrm{d} ; d_{L}$ and $\mathrm{dm}$ respectively.

- $K_{E}$ is the carrying capacity related to the amount of available nutrients and space

- $\mathrm{b}$ is the intrinsic oviposition rate which is proportional to the number of females.

- The egg population becomes larvae at a per capita transfer rate $\mathrm{s}$

- The larvae population becomes mosquito female at a per capita rate $s_{L}$

- The flows from eggs to larvae is regulated by a carrying capacity $K_{L}$ due to the intra-specific competition with young larvae.

The mosquito population dynamics is described by:

$$
\left\{\begin{array}{c}
\frac{d E(t)}{d t}=b A(t)\left(1-\frac{E(t)}{K_{E}}\right)-(s+d) E(t) \\
\frac{d L(t)}{d t}=s E(t)\left(1-\frac{L(t)}{K_{L}}\right)-\left(s_{L}+d_{L}\right) L(t) \\
\frac{d A(t)}{d t}=s_{L} L(t)-d_{m} A(t)
\end{array}\right.
$$

This system is de.ned on the bounded subset of $\square^{3}$

$$
\Delta=\left\{\begin{array}{cc} 
& 0 \leq E(t) \leq K_{E} \\
(E, L, A) / & 0 \leq L(t) \leq K_{L} \\
& 0 \leq A(t) \leq \frac{s_{L}}{d_{m}} K_{L}
\end{array}\right\}
$$

\subsection{Virus transmisson}

Recent research have shown, that a genetic mutation in the chikungunya virus identified in Reunion Island, has facilitated the transmission by Aedes albopictus. Indeed, the extrinsic incubation period was reduced to two days [12]. A human is infected after being bitten by an infected vector, after a delay of four to seven days (incubation period), the human is able to transmit the virus. This period, which can go on five to seven days, is the viraemia period. After this time, human recovers [5].

We consider $N_{H}$ the total number of population which we assume constant. We also denote by $b_{H}$ and $d_{H}$ respectively, the human birth and natural death rates. As the virus is transmitted to the human population, we consider an SIR model for the dynamics of this population, where $S_{H}, I_{H}{ }^{a n d} R_{H}$ denote the total number of respectively susceptible, infective, and immune in the human population, we also let $S_{m}$ andI $m$ be the total number of susceptible and infective mosquitoes. The immune class in the vector population does not exist, since mosquitoes carry the infection throughout their life [5].

The effective contact rate $\beta_{H}$ is the average number of contacts per day which would result in infection if the vector is infectious and, as in [13]. The effective contact rate $\beta_{m}$ is the average number of contacts per day that effectively transmit the infection to vectors.

The dynamics that describes the virus transmission to human population is given by:

$$
\left\{\begin{array}{c}
\frac{d S_{H}(t)}{d t}=b_{H} N_{H}-\beta_{H} \frac{\mathrm{I}_{\mathrm{m}}(t)}{A(t)} S_{H}-d_{H} S_{H}(t) \\
\frac{d I_{H}(t)}{d t}=\beta_{H} \frac{\mathrm{I}_{\mathrm{m}}}{A(t)} S_{H}-\left(\gamma+d_{H}\right) I_{H}(t) \\
\frac{d R_{H}(t)}{d t}=\gamma I_{H}(t)-d_{H} R_{H}(t) \\
\frac{d S_{m}(t)}{d t}=s_{L} L(t)-d_{m} S_{m}(t)-\beta_{m} \frac{\mathrm{I}_{H}(t)}{N_{H}} S_{m}(t) \\
\frac{d I_{m}(t)}{d t}=\beta_{m} \frac{\mathrm{I}_{H}(t)}{N_{H}} S_{m}(t)-d_{m} I_{m}(t)
\end{array}\right.
$$

All parameters in this model are positifs.

\section{A MODEL FOR OPTIMAL CONTROL}

The model we use for our strategy of control combines both the model describing the life cycle of the mosquitoes and the model that defined the dynamics of the virus transmission with the aim to have an interesting optimal control approach. The model is defined by

$$
\left\{\begin{array}{c}
\frac{d E(t)}{d t}=b A(t)\left(1-\frac{E(t)}{K_{E}}\right)-(s+d) E(t) \\
\frac{d L(t)}{d t}=s E(t)\left(1-\frac{L(t)}{K_{L}}\right)-\left(s_{L}+d_{L}\right) L(t) \\
\frac{d A(t)}{d t}=s_{L} L(t)-d_{m} A(t) \\
\frac{d S_{m}(t)}{d t}=s_{L} L(t)-d_{m} S_{m}(t)-\beta_{m} \frac{I_{H}(t)}{N_{H}} S_{m}(t) \\
\frac{d I_{m}(t)}{d t}=\beta_{m} \frac{I_{H}(t)}{N_{H}} S_{m}(t)-d_{m} I_{m}(t) \\
\frac{d S_{H}(t)}{d t}=b_{H} N_{H}-\beta_{H} \frac{I_{m}(t)}{A(t)} S_{H}-d_{H} S_{H}(t) \\
\frac{d I_{H}(t)}{d t}=\beta_{H} \frac{I_{m}}{A(t)} S_{H}-\left(\gamma+d_{H}\right) I_{H}(t) \\
\frac{d R_{H}(t)}{d t}=\gamma I_{H}(t)-d_{H} R_{H}(t)
\end{array}\right.
$$


There are no prospects of a human vaccine or curative antiviral treatment available in a near future. Therefore, the only opportunity of preventing dissemination of Chikungunya virus consists in reducing the vector density and its contacts with humans. People living in an area colonised by Aedes vector mosquitoes should be taught how to prevent and eliminate man-made breeding sites to reduce the overall vector density around their homes and workplaces. They should be informed about personal protective measures to avoid mosquito bites such as wearing long-sleeve shirts and long trousers and using repellent on exposed skin. Travellers should strictly observe the recommendations for personal protection against mosquito bites while visiting areas where Chikungunya virus transmission is active as cited in [14]. The strategy of the control we adopt consists of both prevention program and free terminal time $t_{f}$. So, we introduce into the model (4) three controls $u_{1}(t), u_{2}(t), u_{3}(t)$ given by

- The first control $u_{1}$ represents efforts made for prevention on a time interval $\left[0, t_{f}\right]$. It mainly consists in reducing the number of vector-host contacts due to the use of repulsive against adult mosquitoes and protection with mosquito nets or wearing appropriate clothing. Indeed Aedes albopictus has a peak of activity during fresh temperatures, early in the morning and late in the afternoon [5].

- The second control $u_{2}$ represents the effect of interventions used for the vector control. It mainly consists in the reduction of breeding sites with chemical application methods, for instance using larvicides like BTI (Bacillus Thuringensis Israelensis) which is a biological larvicide, or by introducing larvivore fish. This control focuses on the reduction of the number of larvae, and thus eggs, of any natural or artificial water-filled container. Moreover, in France, one other type of intervention is the use of traps. This consists in using simple black buckets (black colour is recognized as being attractive), with a capacity of one liter of water, three-quarters full with tannic water (water macerated for 3 days with dead branches and leaves). These traps contain laying sites (little plates of square extruded polystyrene placed on the surface of the water [15].

- The third control $u_{3}$ represents efforts made for treatment on a time interval $\left[0, t_{f}\right]$. It mainly consists in isolating infected patients in hospitals, installingan anti-mosquito electric diffuser in the hospital room, or symptomatic treatments. Because, there is no vaccine or completely satisfying drug to treat all symptoms [11], which can persist several months after the infection [35], the vector control remains a major tool to prevent and control the illness. More precisely, only symptomatic treatments are used in order to alleviate the symptoms. Their efficacy varies from one person to another, using for instance corticosteroids, paracetamol and non-steroidal anti-inflammatory drugs.
Therefore, our transmission and optimal control model of chikungunya disease reads as

$$
\left\{\begin{array}{c}
\frac{d E(t)}{d t}=b A(t)\left(1-\frac{E(t)}{K_{E}}\right)-\left(s+d+\varepsilon u_{2}(t)\right) E(t) \\
\frac{d L(t)}{d t}=s E(t)\left(1-\frac{L(t)}{K_{L}}\right)-\left(s_{L}+d_{L}\right) L(t)-d_{c} u_{2}(t) L(t) \\
\frac{d A(t)}{d t}=s_{L} L(t)-d_{m} A(t) \\
\frac{d S_{m}(t)}{d t}=s_{L} L(t)-d_{m} S_{m}(t)-\beta_{m} \frac{I_{H}(t)}{N_{H}} S_{m}(t)\left(1-u_{1}(t)\right) \\
\frac{d I_{m}(t)}{d t}=\beta_{m} \frac{I_{H}(t)}{N_{H}} S_{m}(t)\left(1-u_{1}(t)\right)-d_{m} I_{m}(t) \\
\frac{d S_{H}(t)}{d t}=b_{H} N_{H}-\beta_{H} \frac{I_{m}(t)}{A(t)} S_{H}\left(1-u_{1}(t)\right)-d_{H} S_{H}(t) \\
\frac{d I_{H}(t)}{d t}=\beta_{H} \frac{I_{m}}{A(t)} S_{H}\left(1-u_{1}(t)\right)-\left(\gamma+d_{H}+\gamma_{0} u_{3}(t)\right) I_{H}(t) \\
\frac{d R_{H}(t)}{d t}=\gamma I_{H}(t)-d_{H} R_{H}(t)
\end{array}\right.
$$

\section{THE OPTIMAL CONTROL PROBLEM}

The first control considered $u_{1} \in[0,1]$ and corresponds to prevention effort, thus if $u_{1}=1$ there is no contact between humans and mosquitoes and if $\mathrm{u} 1=0$ the infection rate is maximal and equal to $\beta_{H}$ or $\beta_{m}$

The second control $u_{2} \in[0,1]$ corresponds to the treatment efforts and 0 is the proportion of effective treatment (thus $u_{2}(t)$ is the per capita recovery rate induced by treatment).

The third control $u_{3} \in[0,1]$ corresponds to the reduction of the mosquito proliferation effort, and $\varepsilon$ and $d_{c}$ are eggs and larvae mortality rates induced by chemical intervention respectively.

We consider the objective functional for our control problem given by

$J\left(u, u_{2}, u_{3}, t\right)=\int_{0}^{t_{t}} I_{u}(t)+I_{n}(t)+L(t)-R_{H}(t)+B u_{1}^{2}(t)+B_{z_{2}} u_{2}^{2}(t)+B u_{3}^{2}(t) d t+t^{2}$

(6)

Positive constants $B_{1}, B_{2}$ and $B_{3}$ are weight for prevention, treatment and vector control effort respectively, which regularize the optimal control. Where $t_{f}$ represents the duration of the vaccination program. Our goal is to minimize the duration of our optimal control approach and systemic costs attempting to reduce the number of infected human and mosquitoes, and Larvae group and also increase the removed individuals. We seek an optimal control $u_{1}^{*}, u_{2}^{*} ; u_{3}{ }_{3}$ and an optimal terminal time $t_{f}^{*}$ such that

$J\left(u_{1}{ }^{*}, u_{2}{ }_{2}, u_{3}{ }_{3}, t^{*},{ }^{*}\right)=\min \left\{J\left(u_{1}, u_{2}, u_{3}, t_{f}\right):\left(u_{1}, u_{2}, u_{3}\right) \in U, t_{f} \in \square^{+}\right\}$

Where $\mathrm{U}$ is the set of admissible controls defined by

$U=\left\{u(t): 0 \leq u \leq b, \mathrm{t} \in\left[0, t_{f}\right]\right\}$ 
Where $\mathrm{u}(\mathrm{t})$ is a piecewise function on $\left[0, t_{f}\right]$

The basic framework of this problem is to prove the following

- The existence of the optimal control.

- The characterization of the optimal control.

- The characterization of the optimal final time needed to reduce the human population and limit the mosquitoes compartment.

The first framework which is to prove the existence of the optimal control is done in [5], where the following properties are verified:

1. The set of controls and corresponding state variables is non empty;

2. The control set $U$ is convex and closed;

3. The right hand side of the state system is bounded by a linear function in the state and control;

4. The integrand of the objective functional is convex;

5. There exist constants $c_{1}, c_{2}, c_{3} \succ 0$, and $\beta \succ 0$ such that the integrand of the objective functional is bounded below by

$$
c_{1}\left(\left|u_{1}\right|^{2}+\left|u_{2}\right|^{2}+\left|u_{3}\right|^{2}\right)^{\frac{\beta}{2}}-c_{2}
$$

Pontryagin's Maximum Principal converts (5), (6) and (7) into a problem of minimizing a Hamiltonian, defined by

$$
H=I_{H}+I_{m}+L-R_{H}+B_{1} u^{2}{ }_{1}+B_{2} u^{2}{ }_{2}+B_{3} u^{2}{ }_{3}+\sum_{i=1}^{3} \lambda_{i} f_{i}
$$

where $f_{i}$ is the right side of the differential equation of the $i^{\text {th }}$ state variable.

By applying the Pontryagin's maximum principle [10], we obtain the following theorem

Theorem1:

Given an optimal control $u^{*}$ an optimal terminal time $t_{f}^{*}$, and solutions $E^{*}, L^{*}, A^{*}, S_{m}^{*}, I^{*}, S_{H}^{*}, I_{H}^{*}$ and $\mathrm{R}_{H}^{*}$ of the corresponding state system, there exists an adjoint vector $\lambda=\left[\lambda_{1}, \lambda_{2}, \lambda_{3}, \lambda_{4}, \lambda_{5}, \lambda_{6}, \lambda_{7}, \lambda_{8}\right]$ satisfying

$$
\begin{gathered}
\frac{d \lambda_{1}(t)}{d t}=\lambda_{1}(t)\left(\frac{b A(t)}{K_{E}} s+d+\varepsilon u_{2}(t)\right)+\lambda_{2} s\left(\frac{L(t)}{K_{L}}-1\right) \\
\frac{d \lambda_{2}(t)}{d t}=-1+\lambda_{2}\left(\frac{s E(t)}{K_{L}}+s_{L}+d_{L}+d_{c} u_{2}(t)\right)-s_{L}\left(\lambda_{3}+\lambda_{4}\right) \\
\frac{d \lambda_{3}(t)}{d t}=\lambda_{1} b\left(\frac{E(t)}{K_{E}}-1\right)+\lambda_{3} d_{m}+\beta_{H} \frac{I_{m}(t) S_{H}(t)}{A^{2}(t)}\left(1-u_{1}(t)\right)\left(\lambda_{7}-\lambda_{6}\right) \\
\frac{d \lambda_{4}(t)}{d t}=\lambda_{4} d_{m}+\left(\lambda_{4}-\lambda_{5}\right) \beta_{m} \frac{I_{H}(t)}{N_{H}}\left(1-u_{1}(t)\right) \\
\frac{d \lambda_{5}(t)}{d t}=-1+\lambda_{5} d_{m}+\beta_{H} \frac{S_{H}(t)}{A(t)} S_{m}(t)\left(1-u_{1}(t)\right)\left(\lambda_{7}-\lambda_{6}\right) \\
\frac{d \lambda_{6}(t)}{d t}=\lambda_{6} d_{H}+\beta_{H} \frac{I_{m}(t)}{A(t)}\left(1-u_{1}(t)\right)\left(\lambda_{6}-\lambda_{7}\right) \\
\frac{d \lambda_{7}(t)}{d t}=-1+\left(\lambda_{4}-\lambda_{5}\right) \beta_{m} \frac{S_{m}(t)}{N_{H}\left(1-u_{1}(t)\right)+\left(\gamma+d_{H}+\gamma_{0} u_{3}(t)\right) I_{H}(t)-\lambda_{8} \gamma} \\
\frac{d \lambda_{8}(t)}{d t}=1+\lambda_{8} d_{H}
\end{gathered}
$$

With the transversality conditions

$\lambda_{1}\left(t_{f}\right)=\lambda_{2}\left(t_{f}\right)=\lambda_{3}\left(t_{f}\right)=\lambda_{4}\left(t_{f}\right)=\lambda_{5}\left(t_{f}\right)=\lambda_{6}\left(t_{f}\right)=\lambda_{7}\left(t_{f}\right)=\lambda_{8}\left(t_{f}\right)=0$
Furthermore, the optimal control $u^{*}$ is given by

The system (10) is defined by

$$
\begin{aligned}
& u_{1}^{*}=\min \left(b, \max \left(0, \frac{\left(\lambda_{5}-\lambda_{4}\right) \beta_{m} \frac{I_{H}}{N_{H}} S_{m}+\left(\lambda_{6}-\lambda_{7}\right) \beta_{H} \frac{I_{m}}{A} S_{H}}{2 B_{1}}\right)\right) \\
& u_{2}^{*}=\min \left(b, \max \left(0, \frac{\lambda_{1} \varepsilon E+\lambda_{2} d_{c} L}{2 B_{2}}\right)\right) \\
& u_{3}^{*}=\min \left(b, \max \left(0, \frac{\lambda_{7} \gamma_{0} I_{H}}{2 B_{3}}\right)\right)
\end{aligned}
$$

The optimal final time is given by

$$
t_{f}^{*}=R_{H}\left(t_{f}^{*}\right)-I_{H}\left(t_{f}^{*}\right)-I_{m}\left(t_{f}^{*}\right)-L\left(t_{f}^{*}\right)-\frac{B_{1}}{2}\left(u_{1}\left(t_{f}^{*}\right)\right)-\frac{B_{2}}{2}\left(u_{2}\left(t_{f}^{*}\right)\right)-\frac{B_{3}}{2}\left(u_{3}\left(t_{f}^{*}\right)\right)
$$

\section{Proof}

The adjoint equations and transversality conditions can be obtained by using Pontryagin's Maximum Principle such that

$$
\begin{array}{ll}
\lambda_{1}=-\frac{\partial H}{\partial E} \quad, \quad \lambda_{1}\left(t_{f}\right)=0 & \\
\lambda_{2}=-\frac{\partial H}{\partial L}, & \lambda_{2}\left(t_{f}\right)=0 \\
\lambda_{3}=-\frac{\partial H}{\partial A} \quad, \quad \lambda_{3}\left(t_{f}\right)=0 \\
\lambda_{4}=-\frac{\partial H}{\partial S_{m}} \quad, \quad \lambda_{4}\left(t_{f}\right)=0 \\
\lambda_{5}=-\frac{\partial H}{\partial I_{m}} \quad, \quad \lambda_{5}\left(t_{f}\right)=0 \\
\lambda_{6}=-\frac{\partial H}{\partial S_{H}} \quad, \quad \lambda_{6}\left(t_{f}\right)=0 \\
\lambda_{7}=-\frac{\partial H}{\partial I_{H}}, \quad \lambda_{7}\left(t_{f}\right)=0 \\
\lambda_{8}=-\frac{\partial H}{\partial R_{H}} \quad, \quad \lambda_{8}\left(t_{f}\right)=0
\end{array}
$$

The optimal control $u_{1}^{*}, u_{2}^{*}, u_{3}^{*}$ can be solve from the optimality condition,

$$
\frac{\partial H}{\partial u_{1}}=0, \frac{\partial H}{\partial u_{2}}=0, \frac{\partial H}{\partial u_{3}}=0
$$

That is

$\frac{\partial H}{\partial u_{1}}=2 \mathrm{~B}_{1}+\left(\lambda_{4}-\lambda_{5}\right) \beta_{m} \frac{I_{H}}{N_{H}} \mathrm{~S}_{m}+\left(\lambda_{7}-\lambda_{6}\right) \beta_{H} \frac{I_{m}}{A} \mathrm{~S}_{H}=0$

$\frac{\partial H}{\partial u_{2}}=2 \mathrm{~B}_{2}-\lambda_{1} \varepsilon \mathrm{E}-\lambda_{2} \mathrm{~d}_{c} \mathrm{~L}=0$

$\frac{\partial H}{\partial u_{3}}=2 \mathrm{~B}_{3}-\lambda_{7} \gamma_{0} \mathrm{I}_{H}=0$

By the bounds in the conrol $\mathrm{U}$, it is easy to obtain $\left(u_{1}^{*}, u_{2}^{*}, u_{3}^{*}\right)$ in the form of (10).

The transversality condition for $t_{f}$ o be the optimal terminal time can be stated as 
$H\left(t_{f}^{*}, x\left(t_{f}^{*}\right), \lambda\left(t_{f}^{*}\right), u\left(t_{f}^{*}\right)\right)=-\frac{\partial g}{\partial t}\left(t_{f}^{*}, x\left(t_{f}^{*}\right)\right)$

Where

$$
g(t, x(t))=t_{f}^{2}
$$

That is

$$
-R_{H}\left(t_{f}^{*}\right)+I_{H}\left(t_{f}^{*}\right)+I_{m}\left(t_{f}^{*}\right)+L\left(t_{f}^{*}\right)+\frac{B_{1}}{2}\left(u_{1}\left(t_{f}^{*}\right)\right)+\frac{B_{2}}{2}\left(u_{2}\left(t_{f}^{*}\right)\right)+\frac{B_{3}}{2}\left(u_{3}\left(t_{f}^{*}\right)\right)=2 t_{f}
$$

Thus, $t_{f}$ may be rewritten as in (13)

\section{NUMERICAL SIMULATIONS}

We consider a free terminal time optimal control problem, because of the complex structure of Hamiltonian systems, many iterations are needed to solve a free terminal control problem due to the transversality condition (14) for the terminal time. In this section we present the results obtained by solving numerically the optimality system. This system consists of a two-point boundary value problem, with separated boundary conditions at times $\mathrm{t}=0$ and $\mathrm{t}=t_{f}$.

There were initial conditions for the state variables and terminal conditions for the adjoints. In addition, the final time $t_{f}$ itself is now a variable and must satisfy the transversality condition (13). The algorithm we used proceeds as follows

\section{Algorithm 2}

- Start with an initial guess of the final time;

- Choose initial guess of the state variables, the adjoint variables and the control;

- Forward solving of the state system;

- Backward solving of the adjoint system ;

- Update the control using the characterization (10);

- Update the terminal time using the characterization (13);

- Continue until the optimality condition is achieved.

The state (5) is solved forward in time with initial conditions $\mathrm{Z}(0)=(100 ; 40 ; 10 ; 0: 9 ; 0: 1 ; 0: 2)$ while the adjoint (or costate) system( 14$)$ is solved backward in time with terminal conditions $(\mathrm{T})=(0 ; 0 ; 0 ; 0 ; 0 ; 0)$, where $\mathrm{T}=100$ days.

For a detailed description of the semi-implicit Finite difference method used for solving the optimality system, we refer the interested reader to [7]. The numerical simulations are carried out using Matlab and using the following parameter values and initial conditions taken for [5].

Table1

\begin{tabular}{|l|l|l|}
\hline Parameter & Description & Value \\
\hline $\mathrm{b}$ & per capita oviposition rate & 1 or 6 \\
\hline$K_{E}$ & carrying capacity for eggs & 1000 \\
\hline$\varepsilon$ & Chemical eggs mortality rate & 0.001 \\
\hline$K_{L}$ & Carrying capacity for larvae & 500 \\
\hline$S$ & Transfer rate from eggs to larvae & 0.7 \\
\hline$s$ & $\begin{array}{l}\text { Transfer rate from Larvae to } \\
\text { mosquitoes }\end{array}$ & 0.5 \\
\hline$d$ & Eggs death rate & 0.2 or 0.4 \\
\hline
\end{tabular}

\begin{tabular}{|l|l|l|}
\hline$d_{L}$ & Larvae natural mortality rate & 0.2 or 0.4 \\
\hline$d_{c}$ & Chemical larvae mortality rate & 0.3 \\
\hline$d_{m}$ & Adult mosquitoes mortality rate & $0.25 \quad$ or \\
& \multicolumn{2}{|c|}{0.5} \\
\hline$b_{H}$ & Human birth rate & 0.0000457 \\
\hline$\beta_{H}$ & $\begin{array}{l}\text { Effective contact rate human - } \\
\text { >vector }\end{array}$ & $0.2 \quad$ or \\
\hline$\beta_{m}$ & $\begin{array}{l}\text { Effective contact rate vector - } \\
>\text { human }\end{array}$ & 0.1 or 0.5 \\
\hline$\gamma_{H}$ & Natural recovery rate & 0.1428 \\
\hline
\end{tabular}

- $\quad$ Since the mosquito population dynamic and the virus transmission dynamics and control functions are on different scales. The other parameters are given in table 1 .

As defined above, we attempt to give the optimal free final time needed to reduce both the infected group of human and mosquitoes, by this way, numerical simulations suggest 38 days as final time of the approach.

The eggs of Aedes aegypti are smooth, long, ovoid shaped, and roughly one millimeter long. When first laid, eggs appear white but within minutes turn a shiny black. In warm climates eggs may develop in as little as two days, whereas in cooler temperate climates, development can take up to a week. Laid eggs can survive for very long periods in a dry state, often for more than a year, that's why in the stage without control evolution of the number of eggs take higher levels, once controlled, the number of eggs doesn't develop as shown in Figure1.

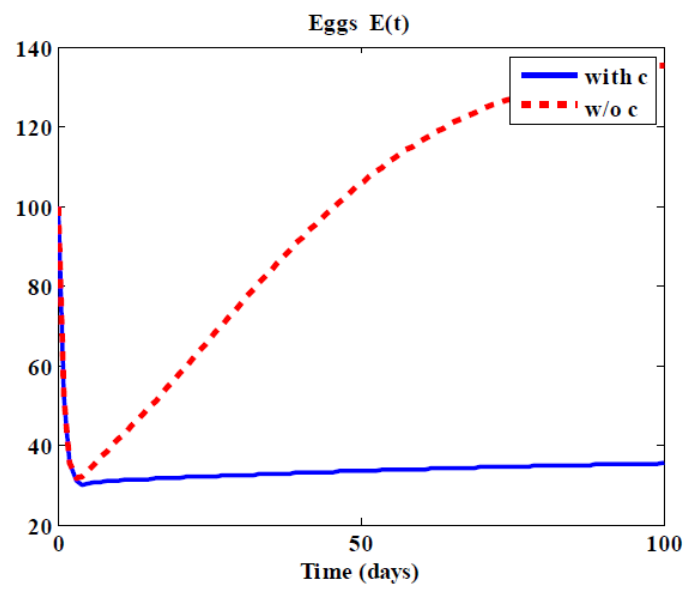

Fig 1: The evolution of Egg group with and without control

After hatching of the eggs, the larvae feed on organic particulate matter in the water, Most of the larval stage is spent at the water's surface, although they will swim to the bottom of the container if disturbed or when feeding. Larvae are often found around the home in puddles, tires, or within any object holding water. Larval development is temperature dependent. The larvae pass through four instars, spending a short amount of time in the first three, and up to three days in the fourth instar. That's why we have the effectiveness of the control used in this stage, which focuses on the reduction of 
the number of larvae of any natural or artificial water-filled container, as shown in Figure2 which presents both the evolution of larvae with and without control.

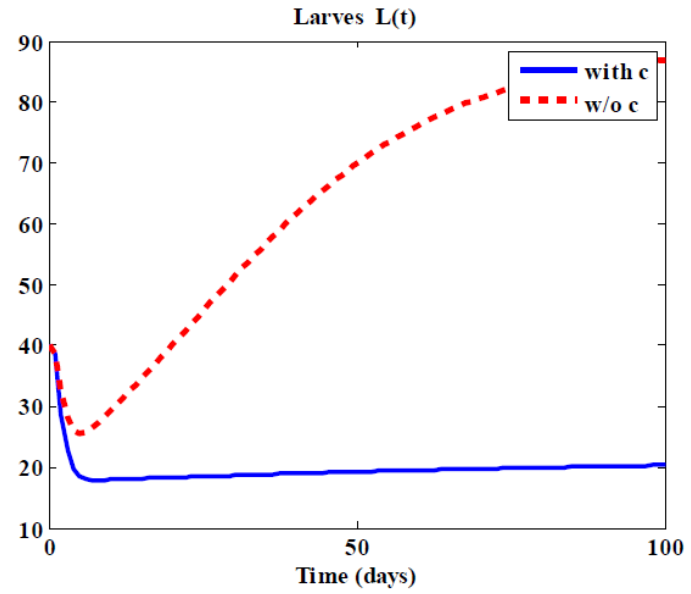

Fig 2: The evolution of Larvae group with and without control

The life expectancy for males is fifteen days whereas it is from two to four weeks for females and can reach ten weeks under labs conditions. In Figure3, we present the evolution of adults with and without control, we can remark that in the case without control, the number of adults mosquitoes increase to high levels, while in the case with control we success to limit this developement, and then reduce the number of infections that can be spread to humans.

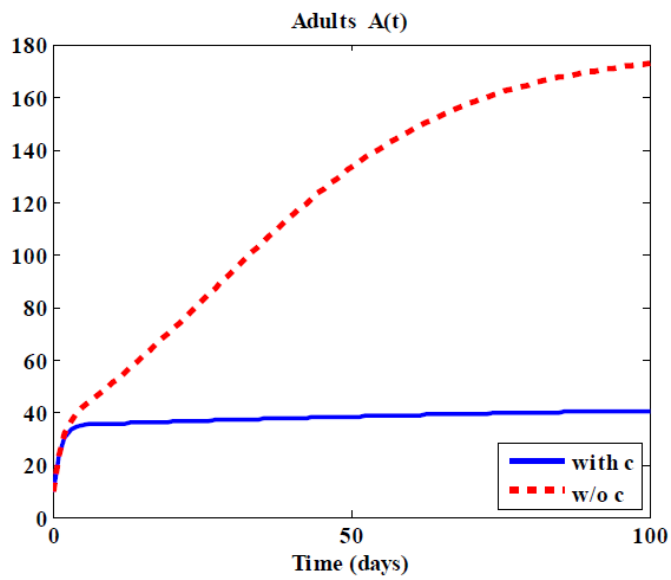

Fig 3: The evolution of Adult group with and without control

As presented in the first parts of this paper, Chikungunya viruses are transmitted to humans through the bites of infective female Aedes mosquitoes. The mosquitoes generally acquire the virus while feeding on the blood of an infected person. But there is no way to tell if a mosquito is carrying the chikungunya virus. Infected female mosquitoes may also transmit the virus to their of spring by transovarial (via the eggs) transmission, but the role of this in sustaining transmission of the virus to humans has not yet been defined. That's why even if our approach successes to limit development of the mosquitoe. In Figure 4, we remark that the number of susceptible human is more important than in the case without control, this is due to the aim of our approach which focuses on the reduction of the number of infected human and vector, and the nature of the virus transmission, so in the case without control the number of adults mosquitoies increase to high levels, then the number of susceptibke person is less, because it's the number of infected humans which increase. While in the case with control, the vector is reduced so most of human are on the case of susceptible persons.

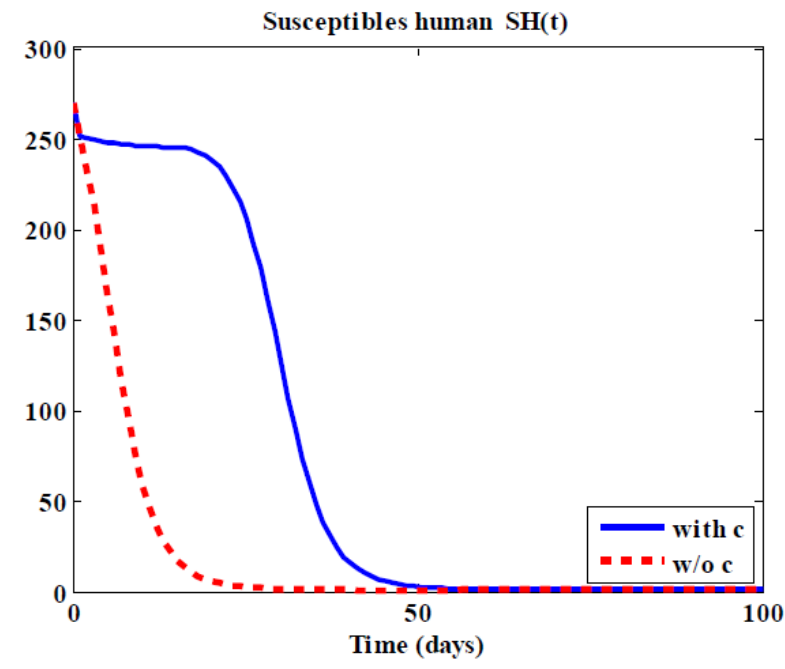

Fig 4: The evolution of susceptible humans with and without control

In Figure5, it's the evolution of infected persons which is presented, the major case of our appr oach, and the graph below shows the effectiveness of the study done in this work. As given above, the numerical simulations suggested 38 days as minimal duration for treatment, the control used in this compartment, mainly consists in isolating infected patients in hospitals, installingan anti-mosquito electric diffuser in the hospital room, or symptomatic treatments. Because, there is no vaccine nor completely satisfying drug to treat all symptoms, so reducing a little number of infected humans can be benefic in order to limit spread of Chikungunya. This difference if evident in Figure5 which presents both the case with and without control of infected human.

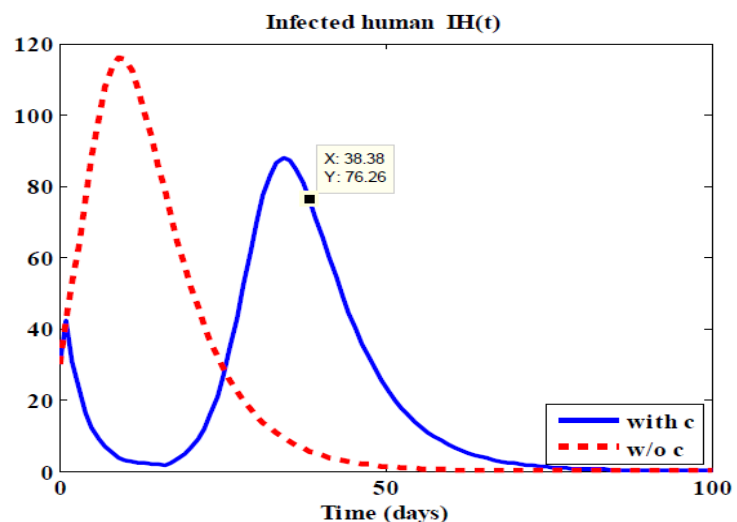

Fig 5: The evolution of Infected Humans with and without control 


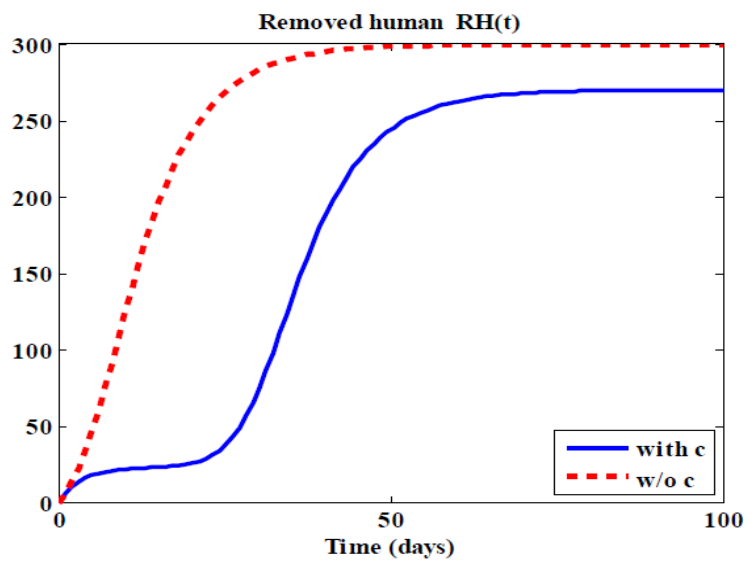

Fig 6: The evolution of removed group with and without control

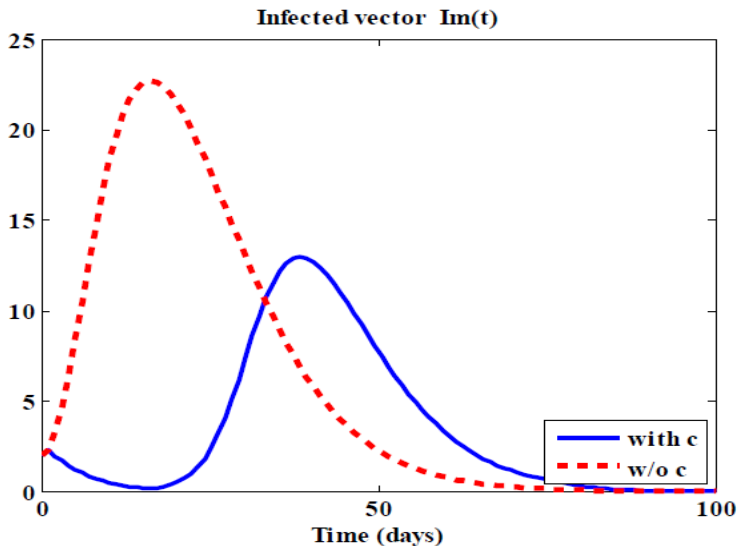

Fig 7: The evolution of infected mosquitoe group with and without control

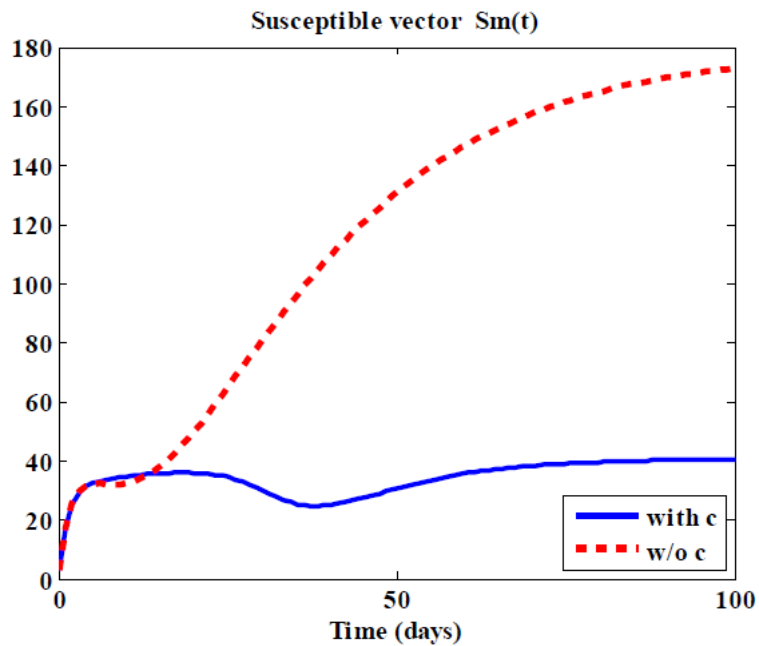

Fig 8: The evolution of susceptible group with and without control

\section{CONCLUSION}

The purpose of this work is to derive a new control strategy for an SIR epidemic model, considering a control simulating vaccination program. By this way, both the terminal time, and a control $\mathrm{u}$ representing the rate of susceptible individuals being vaccinated per time unit, were aim to minimize. Numerical simulations proved an interesting result : The infected group and the terminal time are reduced, which confirm the effectiveness of the approach.

As perspective of this work we are trying to turn new numerical simulations in order to give best reduction of infected human and eradicate definitively the disease.

\section{ACKNOWLEDGEMENT}

The authors acknowledge with appreciation the assistance offered by the Moroccan Systems Network Theory for supporting the research reported in this paper. They express also their gratitude to the entire staff.

\section{REFERENCES}

[1] Rudolph KE, Lessler J, Moloney RM, Kmush B,Cummings DA. Incubation periods of mosquito-borne viral infections: a systematic review. Am J Trop Med Hyg. 2014 May;90(5):882-91

[2] Van Bortel W, Dorleans F, Rosine J, Blateau A, Rousset $\mathrm{D}$, Matheus S, et al. Chikungunya outbreak in the Caribbean region, December 2013 to March 2014, and the signi.cance for Europe. Euro Surveill. 2014;19(13).

[3] European Centre for Disease Prevention and Control.Aedes albopictus factsheet [Internet]. Available from

http://www.ecdc.europa.eu/en/healthtopics/vectors/mosq uitoes/Pages/aedes-albopictus-factsheet.aspx.

[4] Alkama, M., et al. "Free terminal Time OptimalControlProblem of an SIR Epidemic Model with Vaccination."2014, International Journal of Science andResearch, ISSN 2319-7064.Research, ISSN 23197064.

[5] Moulay, D., M. A. Aziz-Alaoui, and M. Cadivel. "Thechikungunya disease: modeling, vector andtransmissionglobal dynamics." Mathematical biosciences229.1 (2011): 50-63.

[6] Moulay, Djamila, M. A. Aziz-Alaoui, and Hee-Dae Kwon. "Optimal control of chikungunya disease: larvaereduction, treatment and prevention." MathematicalBiosciences and Engineering 9.2 (2012): 369-392.

[7] M. Elhia , O. Balatif , J. Bouyaghroumni, E. Labriji, M. Rachik, Optimal Control Applied to the Spread of Inluenza A(H1N1), Applied Mathematical sciences, Vol.6, 2012, no. 82, 4057.4065

[8] E. Jung, S. Lenhart and Z. Feng, Optimal control of treatments in a two-strain tuberculosis model, Discrete and Continuous Dynamical Systems Series B, 2 (2002), 473482 .

[9] K. Blayneh, Y. Cao and H.D. Kwon, Optimal control of vector-borne disease: Treatment and prevention, Discreteand Continuous Dynamical Systems Series B, 11 (2009), 587.611.

[10] L. S. Pontryagin, V. G. Boltyanskii, R. V. GamkrelidzeE. F. Mishchenko, The Mathematical Theory of Optimal Processes, Wiley, New York, 1962.

[11] H. Delatte, G. Gimonneau, A. Triboire, D. Fontenille,Influence of temperature on immature development, survival, longevity, fecundity, and gonotrophic cycles ofAedes albopictus, vector of chikungunya and dengue in the Indian Ocean, J. Med. Entomol. 46 (2009) 33.41. 
[12] M. Dubrulle, L. Mousson, S. Moutailler, M. Vazeille, A.Failloux, Chikungunya virus and Aedes mosquitoes:saliva is infectious as soon as two days after oral infection, PLoS ONE 4 (6) (2009) e5895.

[13] J.X. Velasco-Hernàndez, A model for chagas disease involving transmission by vectors and blood transfusion, Theor. Popul. Biol. 46 (1) (1994) 1.

[14] Noël, H., and C. Rizzo. "Spread of chikungunya from the Caribbean to mainland Central and South America: a greater risk of spillover in Europe." Euro surveillance: bulletin Européen sur les maladies transmissibles European communicable diseasebulletin 19.28 (2014).

[15] Be dry with mosquitoes. Http://www.albopictus.eid med.org/index.htm, (2011).

[16] Outbreaks, Recent Chikungunya. "Chikungunya: nolonger a third world disease." (2007).

[17] Charrel, Rémi N., Xavier de Lamballerie, and DidierRaoult. "Chikungunya outbreaks-the globalization ofvectorborne diseases." New England Journal ofMedicine 356.8 (2007): 769. 